\title{
Automatic Fault Detection and Identification of Exact Location of the Pin Insulator in Power Distribution Lines
}

\author{
Shubham Ashtekar ${ }^{1}$, Mahesh Kunnurkar ${ }^{2}$ \\ Department of Electrical \& Electronics Engineering, \\ KLS's Gogte Institute of Technology, Belgaum, Karnataka, India
}

\begin{abstract}
One of the recent problems which is encountered and detected in the Electrical Distribution Lines is in the Pin Insulator such as Breaking of Conductor placed on the Pin Insulator, cracks \& damage in the pin insulator due to its exposure to the atmospheric conditions such as humidity, moisture \& bird excretion etc. Detection and Identification of this problem is very difficult for the operators and linemen working because there are so many distribution lines run across consumer localities and a number of pin insulators placed on each pole and there are so many poles in each distribution lines. In Modern day of human life electricity has a vital importance. Consumers of the electricity always demand a power without interruption and fluctuations in it, And it is also the responsibility of the electricity supplying companies and electricity boards across the globe to provide electrical power more efficiently and take care of all the electrical equipments installed on the system and the proper maintenance of the process and equipments so that the efficiency of the equipments and their work life will be maintained. The proposed system will automatically detect the fault in the distribution lines due to conductor breaking on the pin insulator and its exact location with the help of Current Transformer, Analog to Digital Converter and Raspberry-Pi processor then through the cloud all the collected data is sent at a time to the operator directly in the control room and also to the linemen operating into that area.
\end{abstract}

Keywords:- Electrical Distribution Lines, Pin Insulator, Current Transformer, Analog to Digital Converter, Raspberry-Pi Processor, Control Room.

\section{INTRODUCTION}

Insulators are attached to the supports and insulate the conductor from the ground and other conductor material [1]. Normally Pin Insulators are used in electrical power distribution upto $11 \mathrm{KV}$ voltage and sometimes upto $33 \mathrm{KV}$ in primary and secondary distribution of electrical power to the consumers [1]. So it's a necessity for the electricity boards and electrical supply companies to provide the electrical power supply with uninterrupted, efficient and without any fluctuations in it. The main purpose to develop this system is that difficulty faced by the linemen and the operators in detection and identification of the exact location of the fault in distribution lines on the pin insulator. This system will provide detection and exact location identification of the fault in distribution lines. The interruption of current is sensed by the Current Transformer and then the signal is given to the Analog to Digital Converter and Raspberry-Pi Processor after that through cloud the signal is given to operator in the control room and also simultaneously to the linemen operating into that region or area after getting the exact location of the fault the operator can send the linemen directly to the exact location or the linemen into that area goes directly to the location of the fault and repair and maintenance is carried out and the fault is corrected [2].

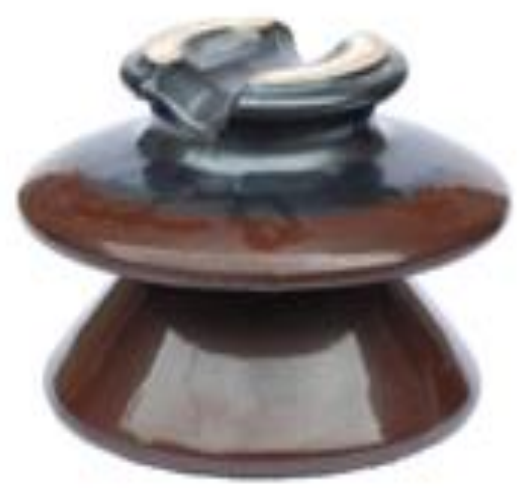

Fig. 1:- Pin type Insulator.

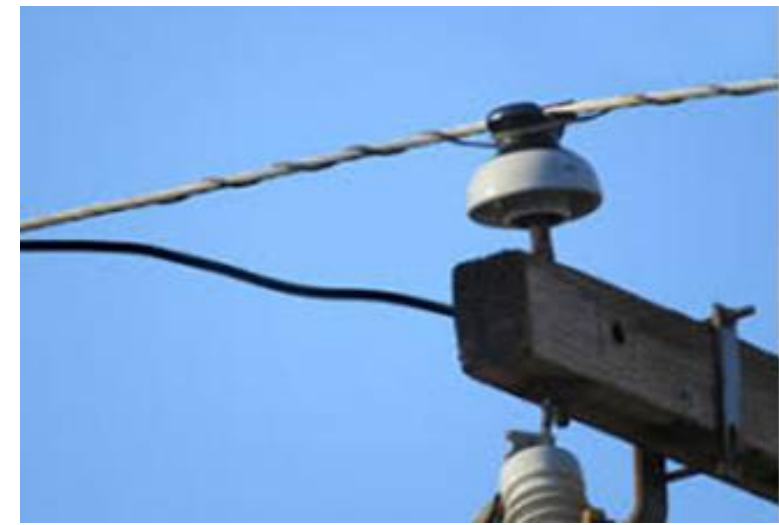

Fig. 2:- Electrical Conductor Placed on a Pin Insulator in the distribution lines. 


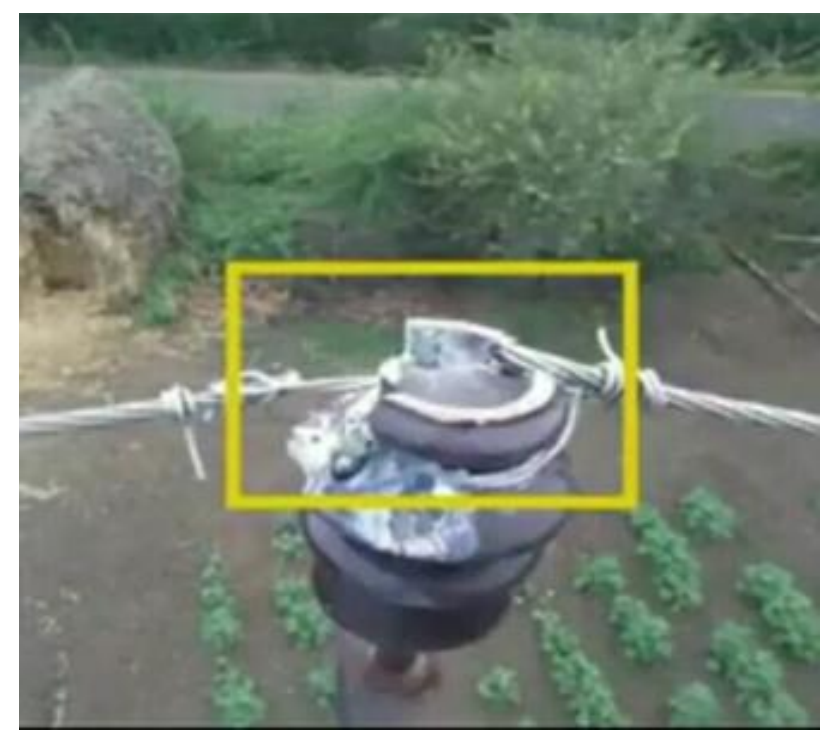

Fig. 3:- shows the condition when the conductor breaks due to the fault.

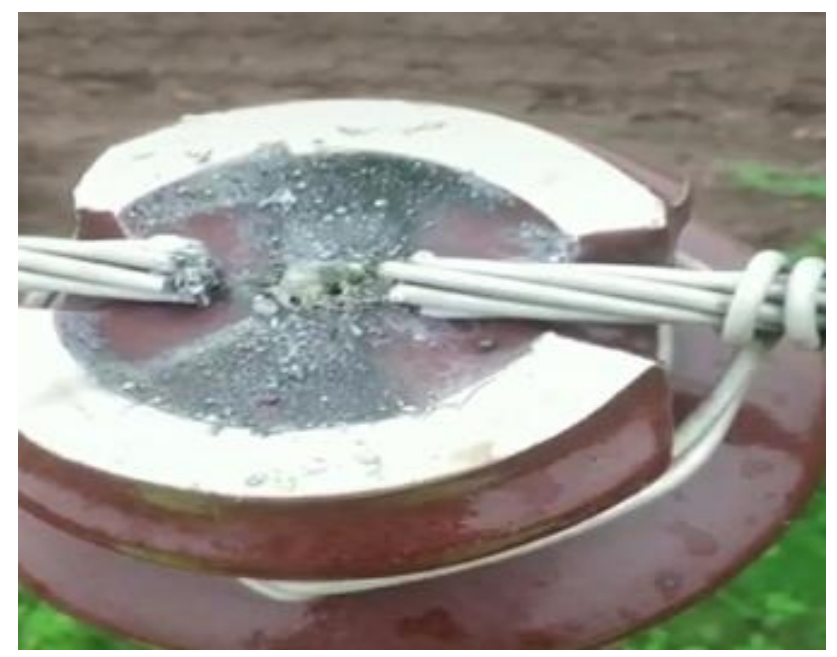

Fig.4:- shows the condition when the damage taking place to the Pin Insulator after fault.

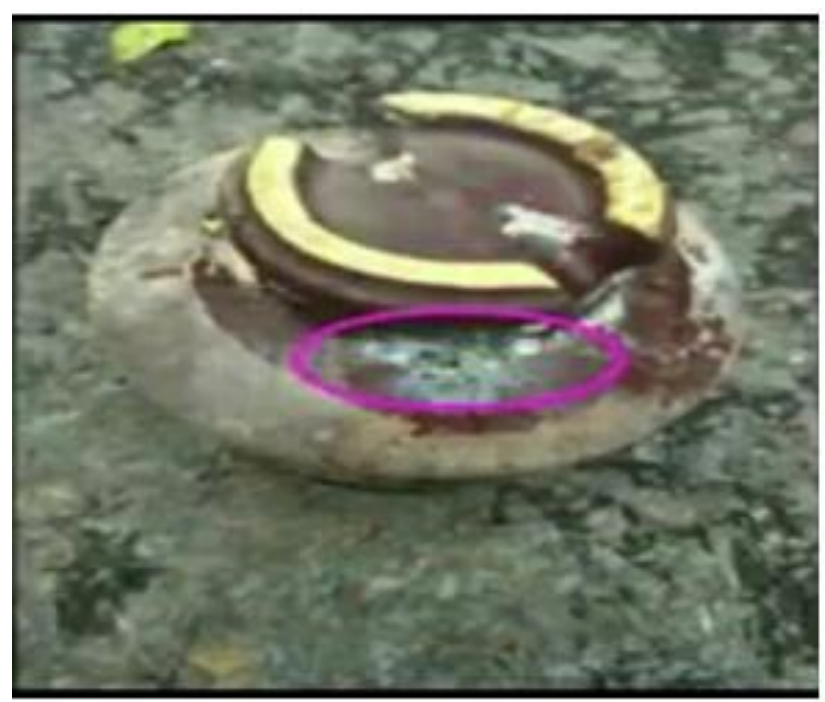

Fig. 5:- shows the condition after the fault when the Pin Insulator is fully damaged due to its exposure to the atmospheric conditions.

\section{AUTOMATIC FAULT DETECTION AND IDENTIFICATION UNIT}

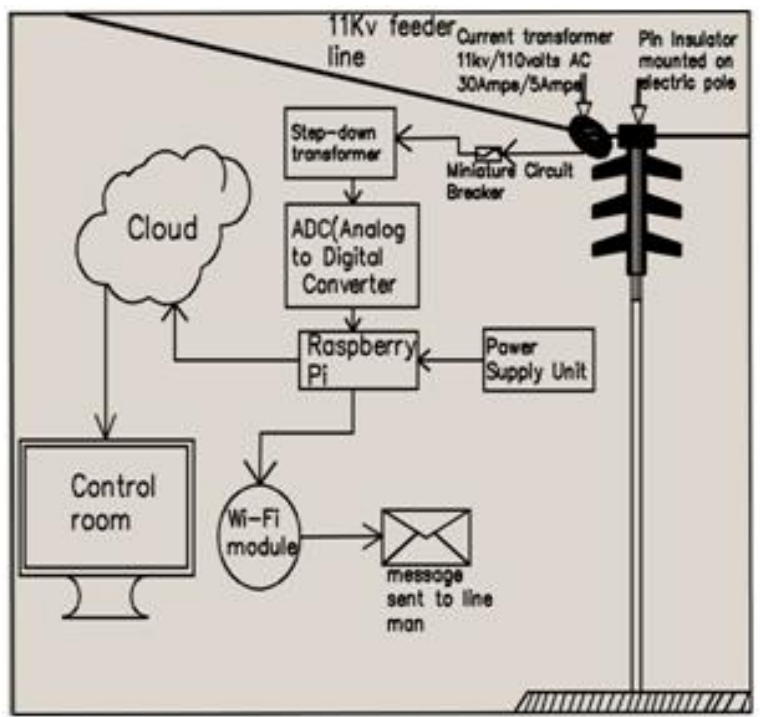

Fig. 1: Block diagram of Automatic Fault Detection and Identification of exact location of the Pin Insulator in Power Distribution Lines.

Figure (1) shows the block diagram of Automatic Fault Detection and Identification unit. Here a Current Transformer (CT) is connected to the $11 \mathrm{KV}$ feeder line of distribution near the electricity pole having a Pin Insulator on it carrying the electrical conductor for power supply. Current Transformer having the ratio 100:1 that is it reduces the voltage level from $11 \mathrm{KV}$ to $100-110 \mathrm{~V}$ and Current from 30A to 5-1 Ampere. Current Transformer will continuously sense the current in the line. Then there is a protective device such as MCB (Miniature Circuit Breaker) used for the protection of low voltage circuit from high voltage circuit. Then the signal is given to the Analog to Digital Converter through a step-down transformer (110V to $5 \mathrm{~V})$. The Analog to Digital Converter is used to convert the $5 \mathrm{~V}$ analog signal to $5 \mathrm{~V}$ digital signal. This digital signal is further passed through a Filter circuit in order to reduce any harmonics present in the signal and then the $5 \mathrm{~V}$ digital signal is given to the Raspberry-Pi Microprocessor which continuously sends signal to the Operator in the Control Room as well as to the mobile phone of the Linemen.

Whenever the fault takes place in the distribution line on the Pin insulator or the breaking of the conductor in the distribution line the Current Transformer will sense that there is break in the line or interruption of the signal, Immediately it will proceed the signal to Analog to Digital Converter from there the signal is given to the Raspberry-Pi Microprocessor. Raspberry-Pi Processor immediately sends the signal through cloud to the Operator operating in the control room and Linemen of that respective area on his respective mobile phone giving them the exact location of the fault. After getting the exact fault location the fault is corrected immediately. Thus saving much of the time of 
identification of fault location and the consumers will get the uninterrupted power supply.

\section{RESULTS}

\author{
import RPI.GPIO as GPIO \\ import time \\ GPIO.setmode(GPIO.BCM) \\ GPIO.setup(5, GPIO.IN) \\ GPIO.setup(6, GPIO.IN) \\ GPIO.setup(13, GPIO.IN) \\ GPIO.setup(19, GPIO.IN)
}

time.sleep(3)

pole1 $=$ GPIO. input (5)

pole $2=$ GPIO.input $(6)$

pole $3=$ GPIO.input $(13)$

pole4 $=$ GPIO.input $(19)$

while True:

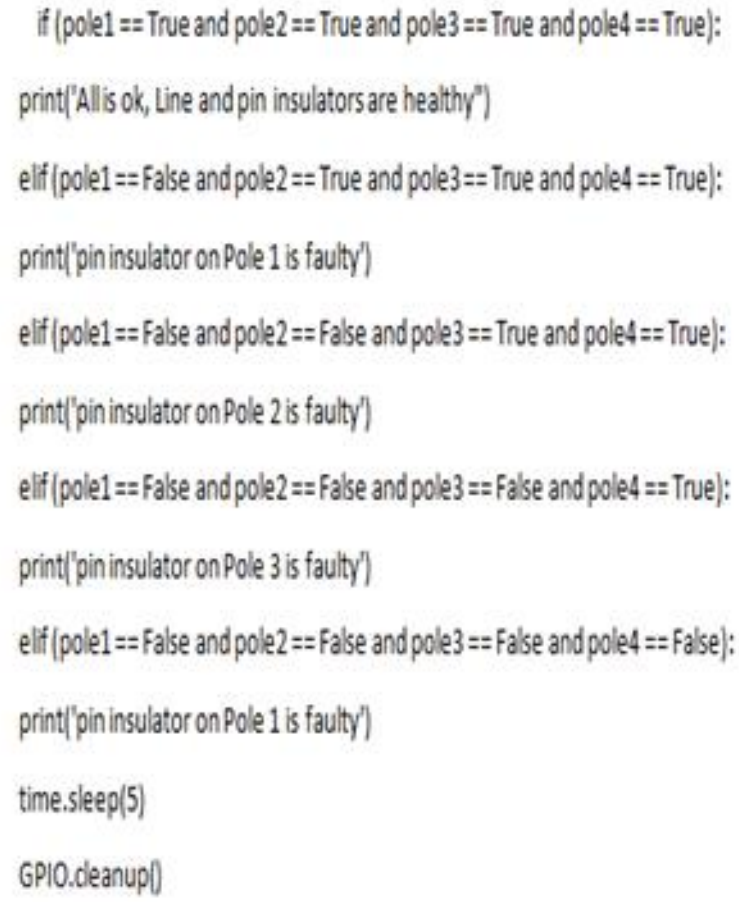

Fig. 1: Program code of the process written in the python programming language in PYCHARM Software of Linux OS which will be implemented in Raspberry-Pi

Microprocessor in the Process.

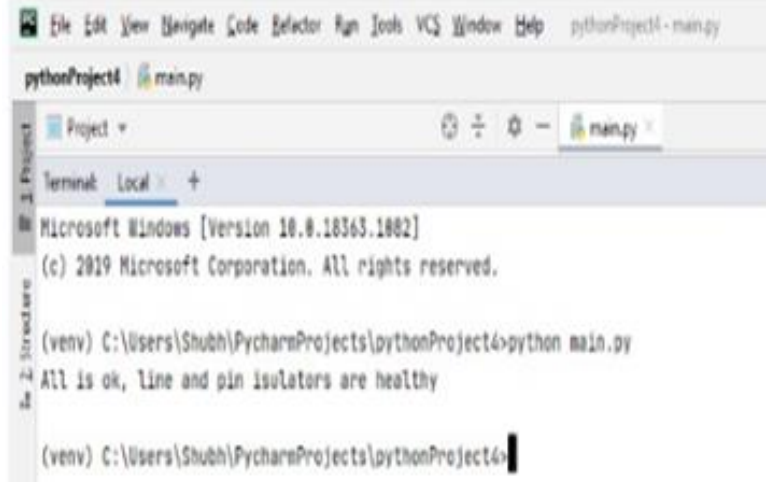

Fig. 2: Program output when all the processes are working properly without any fault in it in observed in the output console window of the PYCHARM Software.

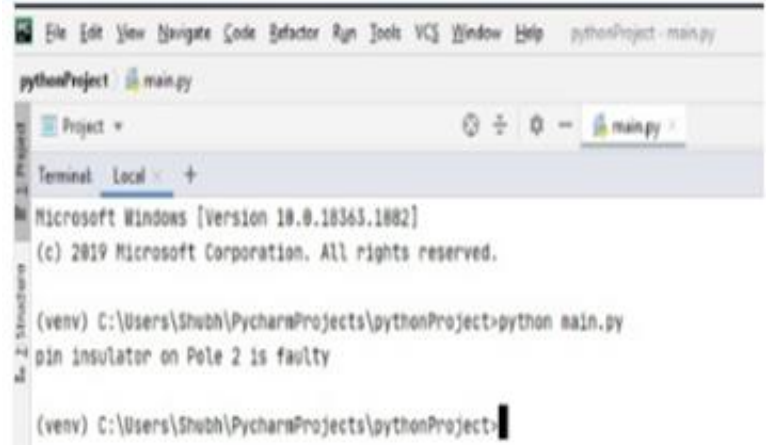

Fig. 3: Program output when the fault occurs in the processes observed in the output console window of the PYCHARM Software.

\section{ADVANTAGES}

Speed of operation is very fast.

The system is highly accurate \& reliable.

- The system is precise to use for detection and identification of fault in the electrical distribution lines.

$>$ System also has provision to send the live data through cloud directly to the operator/linemen's mobile phone and also to the operator in the control room.

$>$ Lots of time will be saved since the faults get rectified very quickly and there will be uninterrupted supply of power to the consumers.

No need of skilled operators.

\section{DISADVATNTAGES}

If there is some malfunction in the Processor, the system may misbehave. But chances of occurrence of such misbehave is almost nil.

> Proper protection and care need to be taken on the system side. 


\section{CONCLUSION}

The Automatic Fault Detection \& Identification of Exact Location of the Pin Insulator in Power Distribution Lines is having several advantages as it provides fast correction of faults in the distribution lines due to Pin Insulator and also provides the exact point of fault to the working operator in the control room and the linemen operating into that region or area, Itself through the cloud and the provisions can be made in the system to get the data directly on the operators or linemen's mobile. So that the faults in the systems are gets rectified as quickly as possible providing consumers an uninterrupted supply of electricity. And also we can improve the whole power scenario. The automatic fault detection and identification of the exact location is achieved by using the Current Transformer, Stepdown Transformer, Analog to Digital Converter and a Raspberry-Pi Microprocessor automatically by sensing the availability of the current in the conductor. And then adjusting the current and voltage to suitable levels and sending the appropriate signal to the operator or controller in the control room directly through the cloud and after that rectifying the faults in the distribution lines.

\section{REFERRENCES}

[1]. Electrical Power Generation, Transmission and Distribution, S. N. Singh, P.H.I., New Delhi, $2^{\text {nd }}$ Edition.

[2]. "Automatic Fault Detection and Location of Transmission Lines using IoT" by Sajal Menon, Don Tommey, Rejoice Thomas Paul, Krishnapriya Vinod, Rajalakshmi Menon.

[3]. Global Research and Development Journal for Engineering, May 2019

[4]. E-ISSN: 2455-5703 